\title{
A Quasi-Spectral Characterization of Strongly Distance-Regular Graphs
}

\author{
M. A. Fiol \\ Departament de Matemàtica Aplicada i Telemàtica \\ Universitat Politècnica de Catalunya \\ Jordi Girona 1-3, Mòdul C3, Campus Nord \\ 08034 Barcelona, Spain; email: fiol@mat.upc.es
}

Submitted: April 30, 2000; Accepted: September 16, 2000.

\begin{abstract}
A graph $\Gamma$ with diameter $d$ is strongly distance-regular if $\Gamma$ is distanceregular and its distance- $d$ graph $\Gamma_{d}$ is strongly regular. The known examples are all the connected strongly regular graphs (i.e. $d=2$ ), all the antipodal distanceregular graphs, and some distance-regular graphs with diameter $d=3$. The main result in this paper is a characterization of these graphs (among regular graphs with $d$ distinct eigenvalues), in terms of the eigenvalues, the sum of the multiplicities corresponding to the eigenvalues with (non-zero) even subindex, and the harmonic mean of the degrees of the distance- $d$ graph.
\end{abstract}

AMS subject classifications. 05C50 05E30

\section{Preliminaries}

Strongly distance-regular graphs were recently introduced by the author [9] by combining the standard concepts of distance-regularity and strong regularity. A strongly distance-regular graph $\Gamma$ is a distance-regular graph (of diameter $d$, say) with the property that the distance- $d$ graph $\Gamma_{d}$ - where two vertices are adjacent whenever they are at distance $d$ in $\Gamma$-is strongly regular. The only known examples of these graphs are the strongly regular graphs (with diameter $d=2$ ), the antipodal distance-regular graphs, and the distance-regular graphs with $d=3$ and third greatest eigenvalue $\lambda_{2}=-1$. (In fact, it has been conjectured that a strongly distance-regular graph is antipodal or has diameter at most three.) For these three families some spectral, or "quasi-spectral", characterizations are known. Thus, it is well-known that strongly regular graphs are characterized, among regular connected graphs, as those having 
exactly three distinct eigenvalues. In the other two cases, however, the spectrum is not enough and some other information about the graphs - such as the numbers of vertices at maximum distance from each vertex - is needed to characterize them. Then we speak about quasi-spectral characterizations, as those in [7, 14] (for antipodal distance-regular graphs) and [5,9] (for strongly distance-regular graphs with diameter $d=3$ ). Quasi-spectral characterizations for general distance-regular graphs were also given in [16,6] (for diameter three) and [10] (for any diameter).

Following these works, we derive in this paper a general quasi-spectral characterization of strongly distance-regular graphs. This includes, as particular cases, most of the previous results about such graphs, and allows us also to obtain some new results about their spectra. In particular, our approach makes clear some symmetry properties enjoyed by their eigenvalues and multiplicities. Eventually, and after looking at the expressions obtained, one gets the intuitive impression that strongly distanceregular graphs are among the graphs with more hidden "spectral symmetries".

Before proceeding to our study we devote the rest of this introductory section to fixing the terminology and to recalling some basic results. Let $\Gamma$ a (simple, finite, and connected) graph with adjacency matrix $\boldsymbol{A}:=\boldsymbol{A}(\Gamma)$ and (set of) eigenvalues ev $\Gamma:=\left\{\lambda_{0}>\lambda_{1}>\cdots>\lambda_{d}\right\}$. The alternating polynomial $P$ of $\Gamma$ is defined as the (unique) polynomial of degree $d-1$ satisfying

$$
P\left(\lambda_{0}\right)=\max _{p \in \mathbb{R}_{d-1}[x]}\left\{p\left(\lambda_{0}\right):\|p\|_{\infty} \leq 1\right\}
$$

where $\|p\|_{\infty}=\max _{1 \leq i \leq d}\left|p\left(\lambda_{i}\right)\right|$. It is known that $P$ is characterized by taking $d$ alternating values \pm 1 at ev $\Gamma: P\left(\lambda_{i}\right)=(-1)^{i+1}, 1 \leq i \leq d$, which, together with Lagrange interpolation, yields

$$
P\left(\lambda_{0}\right)=\sum_{i=1}^{d} \frac{\pi_{0}}{\pi_{i}}
$$

where the $\pi_{i}^{\prime} s$ are moment-like parameters defined from the eigenvalues as $\pi_{i}:=$ $\prod_{j=0, j \neq i}^{d}\left|\lambda_{i}-\lambda_{j}\right|, 0 \leq i \leq d$, and satisfying

$$
\sum_{i \text { even }} \frac{\lambda_{i}^{l}}{\pi_{i}}=\sum_{i \text { odd }} \frac{\lambda_{i}^{l}}{\pi_{i}} \quad(0 \leq l<d) .
$$

(See $[12,14]$ for more details.)

In this work, it is useful to consider the following characterization of distanceregular graphs (see e.g. Biggs [2] or Brouwer et al. [3]): A (connected) graph $\Gamma$, with vertex set $V=\{u, v, \ldots\}$, adjacency matrix $\boldsymbol{A}$, and diameter $d$, is distance-regular if and only if there exists a sequence of polynomials $p_{0}, p_{1}, \ldots, p_{d}$, called the distance polynomials, such that $\operatorname{dgr} p_{i}=i, 0 \leq i \leq d$, and

$$
\boldsymbol{A}_{i}:=\boldsymbol{A}\left(\Gamma_{i}\right)=p_{i}(\boldsymbol{A}) \quad(0 \leq i \leq d),
$$

where $\boldsymbol{A}_{i}$ is the adjacency matrix of the distance- $i$ graph $\Gamma_{i}$ and, so, it is called the distance-i matrix of $\Gamma$. Let $n:=|V|$ and assume that $\Gamma$ has spectrum $\operatorname{sp} \Gamma:=$ 
$\left\{\lambda_{0}^{m\left(\lambda_{0}\right)}, \lambda_{1}^{m\left(\lambda_{1}\right)}, \ldots, \lambda_{d}^{m\left(\lambda_{d}\right)}\right\}$ (as $\Gamma$ is connected, $m\left(\lambda_{0}\right)=1$ ). Then the distance polynomials are orthogonal with respect to the scalar product

$$
\langle p, q\rangle_{\Gamma}:=\frac{1}{n} \operatorname{tr}(p(\boldsymbol{A}) q(\boldsymbol{A}))=\sum_{i=0}^{d} \frac{m\left(\lambda_{i}\right)}{n} p\left(\lambda_{i}\right) q\left(\lambda_{i}\right),
$$

and they are normalized in such a way that $\left\|p_{i}\right\|_{\Gamma}^{2}=p_{i}\left(\lambda_{0}\right), 0 \leq i \leq d$. (Of course, this inner product makes sense - and it will be used later - for any graph $\Gamma$.) Moreover, if $\Gamma_{i}(u)$ denotes the set of vertices at distance $i$ from a given vertex $u$, we have $p_{i}\left(\lambda_{0}\right)=n_{i}(u):=\left|\Gamma_{i}(u)\right|$ for any $u \in V$ and $0 \leq i \leq d$. In fact, there are explicit formulas giving the values of the highest degree polynomial $p_{d}$ at ev $\Gamma$, in terms of the eigenvalues and their multiplicities. Namely,

$$
p_{d}\left(\lambda_{0}\right)=n\left(\sum_{i=0}^{d} \frac{\pi_{0}^{2}}{m\left(\lambda_{i}\right) \pi_{i}^{2}}\right)^{-1} ; \quad p_{d}\left(\lambda_{i}\right)=(-1)^{i} \frac{\pi_{0} p_{d}\left(\lambda_{0}\right)}{\pi_{i} m\left(\lambda_{i}\right)} .
$$

(From the second expression one has the known formulas for the multiplicities in terms of $p_{d}$ - see, e.g., Bannai and Ito [1].) Hence, since $p_{d}\left(\lambda_{0}\right)$ (the degree of $\Gamma_{d}$ ) is a positive integer, the polynomial $p_{d}$ must take alternating values at the mesh ev $\Gamma$ :

$$
p_{d}\left(\lambda_{i}\right)>0 \quad(i \text { even }) ; \quad p_{d}\left(\lambda_{i}\right)<0 \quad(i \text { odd }) .
$$

In this paper, we also use the following results about strongly regular graphs (see, for instance, Cameron's survey [4] or Godsil's textbook [15]):

Lemma 1.1 (a) A graph $\Gamma$, not complete or empty, with adjacency matrix $\boldsymbol{A}$ is $(n, k ; a, c)$-strongly regular if and only if

$$
\boldsymbol{A}^{2}-(a-c) \boldsymbol{A}+(c-k) \boldsymbol{I}=\boldsymbol{J},
$$

where $\boldsymbol{J}$ represents the all-1 matrix.

(b) A connected regular graph $\Gamma$ with exactly three distinct eigenvalues $\mu_{0}>\mu_{1}>$ $\mu_{2}$ is a $(n, k ; a, c)$-strongly regular graph with parameters satisfying

$$
k=\mu_{0}, \quad c-k=\mu_{1} \mu_{2}, \quad a-c=\mu_{1}+\mu_{2} .
$$

Notice that, from the above and $\operatorname{tr} \boldsymbol{A}=0$, it follows that $\mu_{2}<0$ and $\mu_{1} \geq 0$ (with $\mu_{1}=0$ only when $\Gamma$ is the regular multipartite graph).

\section{A quasi-spectral characterization}

In this section we derive our main result, which characterizes strongly-regular graphs among regular graphs, and study some of its consequences.

Given any vertex $u$ of a graph with diameter $d$, we denote by $N_{i}(u), 0 \leq i \leq d$, the $i$-neighbourhood of $u$, or set of vertices at distance at most $i$ from $u$. Using some results from [10,13], the author proved in [8] the following result, which is basic to our study. 
THE ELECTRONIC JOURNAL OF COMBINATORICS 7 (2000), \#R51

Theorem 2.1 Let $\Gamma$ be a regular graph with $n$ vertices and $d+1$ distinct eigenvalues. For every vertex $u \in V$, let $s_{d-1}(u):=\left|N_{d-1}(u)\right|$. Then, any polynomial $R \in \mathbb{R}_{d-1}[x]$ satisfies the bound

$$
\frac{R\left(\lambda_{0}\right)^{2}}{\|R\|_{\Gamma}^{2}} \leq \frac{n}{\sum_{u \in V} \frac{1}{s_{d-1}(u)}}
$$

and equality is attained if and only if $\Gamma$ is a distance-regular graph. Moreover, in this case, we have $\frac{R\left(\lambda_{0}\right)}{\|R\|_{\Gamma}^{2}} R=q_{d-1}:=\sum_{i=0}^{d-1} p_{i}$, where the $p_{i}$ 's are the distance polynomials of $\Gamma$.

Note that the above upper bound is, in fact, the harmonic mean of the numbers $s_{d-1}(u), u \in V$, which is hereafter denoted by $H$. Moreover, in case of equality, $q_{d-1}(\boldsymbol{A})=\sum_{i=0}^{d-1} \boldsymbol{A}_{i}=\boldsymbol{J}-\boldsymbol{A}_{d}$, and the distance- $d$ polynomial of $\Gamma$ is just

$$
p_{d}=q_{d}-q_{d-1}=q_{d}-\frac{R\left(\lambda_{0}\right)}{\|R\|_{\Gamma}^{2}} R
$$

where $q_{d}$ represents the Hoffman polynomial; that is, $q_{d}=\frac{n}{\pi_{0}} \prod_{i=1}^{d}\left(x-\lambda_{i}\right)$.

At this point it is useful to introduce the following notation: For a graph with $n$ vertices, spectrum $\left\{\lambda_{0}^{m\left(\lambda_{0}\right)}, \ldots, \lambda_{d}^{m\left(\lambda_{d}\right)}\right\}$, and alternating polynomial $P$, note that, by (1), the value of $P\left(\lambda_{0}\right)+1$ is given by the sum

$$
\Sigma:=\sum_{i=0}^{d} \frac{\pi_{0}}{\pi_{i}}=1+\Sigma_{e}+\Sigma_{o},
$$

where $\Sigma_{e}$ (respectively $\Sigma_{o}$ ) denotes the sum of the terms $\pi_{0} / \pi_{i}$ with even non-zero (respectively, odd) index $i$. Note also that, by (2) with $l=0$, both numbers are closely related: $\Sigma_{o}=\Sigma_{e}+1$. However, the use of both symbols will reveal the symmetries of the expressions obtained. With the same aim, let us also consider the following sum decomposition:

$$
n=\sum_{i=0}^{d} m\left(\lambda_{i}\right)=1+\sigma_{e}+\sigma_{o},
$$

where $\sigma_{e}$ represents the sum of all multiplicities of the eigenvalues with non-zero even subindex $\sigma_{e}:=m\left(\lambda_{2}\right)+m\left(\lambda_{4}\right)+\cdots$, and $\sigma_{o}:=n-\sigma_{e}-1=m\left(\lambda_{1}\right)+m\left(\lambda_{3}\right)+\cdots$

The following result generalizes for any diameter a theorem of the author [9] (the case of diameter three).

Theorem 2.2 A regular graph $\Gamma$, with $n$ vertices, eigenvalues $\lambda_{0}>\lambda_{1}>\cdots>$ $\lambda_{d}$, and parameters $\sigma_{e}, \Sigma_{e}$ as above, is strongly distance-regular if and only if the harmonic mean of the numbers $s_{d-1}(u), u \in V$, is

$$
H=n-\frac{n \sigma_{e} \sigma_{o}}{n \Sigma_{e} \Sigma_{o}+\left(\sigma_{e}-\Sigma_{e}\right)\left(\sigma_{o}+\Sigma_{o}\right)} .
$$

Moreover, if this is the case, $\Gamma_{d}$ is a strongly regular graph with degree $k=n-H$ and parameters

$$
c=k-k^{2} \frac{\Sigma_{e} \Sigma_{o}}{\sigma_{e} \sigma_{o}} ; \quad a=c+k\left(\frac{\Sigma_{e}}{\sigma_{e}}-\frac{\Sigma_{o}}{\sigma_{o}}\right) .
$$


THE ELECTRONIC JOURNAL of COMBinAtorics 7 (2000), \#R51

Proof. Given any real number $t$, let us consider the polynomial $R:=\left(1+\frac{t}{2}\right) P-\frac{t}{2}$, where $P$ is the alternating polynomial of $\Gamma$. Notice that, from the properties of $P$, we have $R\left(\lambda_{i}\right)=1$ for any odd $i, 1 \leq i \leq d$, and $R\left(\lambda_{i}\right)=-1-t$ for every even $i \neq 0$. Then, the square norm of $R$ satisfies

$$
n\|R\|_{\Gamma}^{2}=R^{2}\left(\lambda_{0}\right)+\sum_{i=1}^{d} R^{2}\left(\lambda_{i}\right) m\left(\lambda_{i}\right)=R^{2}\left(\lambda_{0}\right)+\sigma_{e}(1+t)^{2}+\sigma_{o} .
$$

Hence, by Theorem 2.1, we must have, for any $t$,

$$
\Psi(t):=\frac{n\left[\left(1+\frac{t}{2}\right) P\left(\lambda_{0}\right)-\frac{t}{2}\right]^{2}}{\left[\left(1+\frac{t}{2}\right) P\left(\lambda_{0}\right)-\frac{t}{2}\right]^{2}+\sigma_{e}(1+t)^{2}+\sigma_{o}}=\frac{R\left(\lambda_{0}\right)^{2}}{\|R\|_{\Gamma}^{2}} \leq H .
$$

Since we are interested in the case of equality, we must find the maximum of the function $\Psi$. Such a maximum is attained at

$$
t_{0}=\frac{\sigma_{o}}{\sigma_{e}} \frac{P\left(\lambda_{0}\right)-1}{P\left(\lambda_{0}\right)+1}-1
$$

and its value is

$$
\begin{aligned}
\Psi_{\max }:=\Psi\left(t_{0}\right) & =n-\frac{4 n \sigma_{e} \sigma_{o}}{(n-1) \Sigma^{2}+4 \sigma_{o}\left(\sigma_{e}-\Sigma\right)} \\
& =n-\frac{n \sigma_{e} \sigma_{o}}{n \Sigma_{e} \Sigma_{o}+\left(\sigma_{e}-\Sigma_{e}\right)\left(\sigma_{o}+\Sigma_{o}\right)}
\end{aligned}
$$

where we have used (10) and (11). Consequently, $\Psi_{\max } \leq H$ and, from the same theorem, the equality case occurs if and only if $\Gamma$ is a distance-regular graph with distance $d$-polynomial given by (9). From this fact, let us now see that $\Gamma_{d}$ is strongly regular. In our case, all inequalities in (15) and (14) become equalities with $t=t_{0}$ given by (16), so giving

$$
R\left(\lambda_{0}\right)=\left(1+\frac{t_{0}}{2}\right) P\left(\lambda_{0}\right)-\frac{t_{0}}{2} ; \quad\|R\|_{\Gamma}^{2}=R^{2}\left(\lambda_{0}\right)+\sigma_{e}\left(1+t_{0}\right)^{2}+\sigma_{o} .
$$

This allows us to compute, through (9), the distance- $d$ polynomial and its relevant values:

$$
\begin{aligned}
\mu_{0} & :=p_{d}\left(\lambda_{0}\right)=n-H ; \\
\mu_{1} & :=p_{d}\left(\lambda_{i}\right)=-\frac{R\left(\lambda_{0}\right)}{\|R\|_{\Gamma}^{2}} R\left(\lambda_{i}\right)=\frac{R\left(\lambda_{0}\right)}{\|R\|_{\Gamma}^{2}}\left(1+t_{0}\right) \quad(\text { even } i \neq 0) ; \\
\mu_{2} & :=p_{d}\left(\lambda_{i}\right)=-\frac{R\left(\lambda_{0}\right)}{\|R\|_{\Gamma}^{2}} R\left(\lambda_{i}\right)=-\frac{R\left(\lambda_{0}\right)}{\|R\|_{\Gamma}^{2}} \quad(\text { odd } i) .
\end{aligned}
$$

Then $\Gamma_{d}$ is a regular graph with degree $k=\mu_{0}$ and has at most three eigenvalues $\mu_{0} \geq \mu_{1}>\mu_{2}$ (with multiplicities $m\left(\mu_{0}\right)=1, m\left(\mu_{1}\right)=\sigma_{e}$, and $m\left(\mu_{2}\right)=\sigma_{o}$ ). If it has exactly three eigenvalues, $\mu_{0}>\mu_{1}$, then $\Gamma_{d}$ must be connected and Lemma 1.1 applies. Otherwise, if $\mu_{0}=\mu_{1}$, the graph $\Gamma_{d}$ is simply constituted by disjoint copies 
of the complete graph on $k+1$ vertices. (See [9] for more details.) In both cases $\Gamma_{d}$ is strongly regular, as claimed. Moreover, after some algebraic manipulations, the above formulas yield

$$
\begin{aligned}
k & =\frac{n \sigma_{e} \sigma_{o}}{n \Sigma_{e} \Sigma_{o}+\left(\sigma_{e}-\Sigma_{e}\right)\left(\sigma_{o}+\Sigma_{o}\right)} ; \\
\mu_{1} & =\frac{n \Sigma_{e} \sigma_{o}}{n \Sigma_{e} \Sigma_{o}+\left(\sigma_{e}-\Sigma_{e}\right)\left(\sigma_{o}+\Sigma_{o}\right)}=k \frac{\Sigma_{e}}{\sigma_{e}} ; \\
\mu_{2} & =\frac{-n \sigma_{e} \Sigma_{o}}{n \Sigma_{e} \Sigma_{o}+\left(\sigma_{e}-\Sigma_{e}\right)\left(\sigma_{o}+\Sigma_{o}\right)}=-k \frac{\Sigma_{o}}{\sigma_{o}} ;
\end{aligned}
$$

and the values of $c$ and $a$ follow from (7).

Conversely, if $\Gamma$ is a strongly distance-regular graph, its distance- $d$ graph $\Gamma_{d}$, with adjacency matrix $p_{d}(\boldsymbol{A})$, is a strongly regular graph with either three or two eigenvalues, $\mu_{0} \geq \mu_{1}>\mu_{2}$, satisfying $\mu_{0}=p_{d}\left(\lambda_{0}\right)=n_{d}(u):=\left|\Gamma_{d}(u)\right|$ for every $u \in V$, $\mu_{1}=p_{d}\left(\lambda_{i}\right) \geq 0$ for every even $i \neq 0$, and $\mu_{2}=p_{d}\left(\lambda_{i}\right)$ for every odd $i$. Thus, by adding up the corresponding multiplicities, obtained from the second expression in (4), we get

$$
\sigma_{e}=\sum_{i \text { even }(i \neq 0)} \frac{\pi_{0}}{\pi_{i}} \frac{\mu_{0}}{\mu_{1}}=\frac{\mu_{0}}{\mu_{1}} \Sigma_{e} ; \quad \sigma_{o}=-\sum_{i \text { odd }} \frac{\pi_{0}}{\pi_{i}} \frac{\mu_{0}}{\mu_{2}}=-\frac{\mu_{0}}{\mu_{2}} \Sigma_{o} .
$$

These two equations give the values of $\mu_{1}$ and $\mu_{2}$ - compare with (20) and (21) which, substituted into

$$
n\left\|p_{d}\right\|_{\Gamma}^{2}=\mu_{0}^{2}+\sigma_{e} \mu_{1}^{2}+\sigma_{o} \mu_{2}^{2}=n \mu_{0},
$$

and solving for $\mu_{0}=n_{d}(u)$, give

$$
n_{d}(u)=\frac{n \sigma_{e} \sigma_{o}}{n \Sigma_{e} \Sigma_{o}+\left(\sigma_{e}-\Sigma_{e}\right)\left(\sigma_{o}+\Sigma_{o}\right)}
$$

for every vertex $u \in V$. Hence, all the numbers $s_{d-1}(u)=n-n_{d}(u), u \in V$, are the same and their harmonic mean $H$ satisfies (12). This completes the proof of the theorem.

From the above proof, notice that, alternatively, we can assert that the regular graph $\Gamma$ is strongly distance-regular if and only if the distance- $d$ graph $\Gamma_{d}$ is $k$-regular with degree $k=n_{d}(u)$ given by (19) or (22).

As a by-product of such a proof, we can also give bounds for the sum of "even multiplicities" $\sigma_{e}$ in any regular graph, in terms of its eigenvalues and harmonic mean $H$.

Corollary 2.3 Let $\Gamma$ be a regular graph, with $n$ vertices, eigenvalues $\lambda_{0}>\lambda_{1}>$ $\cdots>\lambda_{d}$, and harmonic mean $H$. Set $\alpha:=\frac{n}{2}\left(1-\frac{1}{H}\right)$ and $\beta:=\Sigma_{e}\left(\frac{n}{H}-1\right)$. Then the sum $\sigma_{e}$ of even multiplicities satisfies the bounds

$$
\alpha-\beta-\sqrt{\alpha(1-\alpha \beta \Sigma)} \leq \sigma_{e} \leq \alpha-\beta+\sqrt{\alpha(1-\alpha \beta \Sigma)} .
$$


Proof. Solve for $\sigma_{e}$ the inequality

$$
n-\Psi_{\max }=\frac{4 n \sigma_{e}\left(n-\sigma_{e}-1\right)}{(n-1) \Sigma^{2}+4\left(n-\sigma_{e}-1\right)\left(\sigma_{e}-\Sigma\right)} \geq n-H,
$$

which comes from (17), (11), and $\Psi_{\max } \leq H$, and use (10).

Let us now consider some particular cases of the above theorem, which contain some previous results of Van Dam, Garriga, and the author. Note first that, for some particular cases, we do not need to know the multiplicity sum $\sigma_{e}$ (and hence nor $\sigma_{o}$ ), since it can be deduced from the eigenvalues. For instance, for diameter $d=3$, the multiplicities $m_{1}, m_{2}, m_{3}$ of a regular graph must satisfy the system

$$
\begin{array}{ccc}
1+m_{1}+m_{2}+m_{3} & = & n \\
\lambda_{0}+m_{1} \lambda_{1}+m_{2} \lambda_{2}+m_{3} \lambda_{3} & = & 0 \\
\lambda_{0}^{2}+m_{1} \lambda_{1}^{2}+m_{2} \lambda_{2}^{2}+m_{3} \lambda_{3}^{2} & = & n \lambda_{0}
\end{array}
$$

whence we get

$$
\sigma_{e}=m_{2}=\frac{\pi_{0}-n\left(\lambda_{1} \lambda_{3}+\lambda_{0}\right)\left(\lambda_{0}-\lambda_{2}\right)}{\pi_{2}} .
$$

Thus, Theorem 2.2 gives the following result.

Corollary 2.4 A connected $\delta$-regular graph $\Gamma$ with $n$ vertices and eigenvalues $\lambda_{0}(=\delta)>\lambda_{1}>\lambda_{2}>\lambda_{3}$ is strongly distance-regular if and only if the harmonic mean of the numbers $s_{2}(u)=1+\delta+n_{2}(u), u \in V$, satisfies

$$
H=n-\frac{4 n \sigma_{o} \sigma_{e}}{(n-1) \Sigma^{2}+4 \sigma_{o}\left(\sigma_{e}+1-\Sigma\right)},
$$

where $\sigma_{e}$ is given by (23), $\sigma_{o}=n-\sigma_{e}-1$ and $\Sigma=\sum_{i=0}^{3}\left(\pi_{0} / \pi_{i}\right)$.

The above result slightly improves a similar characterization given in [9], in terms of the degree of the graph $\Gamma_{3}$, and where the additional condition $\lambda_{2}=-1$ was required.

Let us now consider the case $a=c$. In this case, an strongly regular graph satisfying this condition is also called an $(n, k, c)$-graph (see Cameron [4]) and, consequently, we will speak about an $(n, k, c)$-strongly distance-regular graph.

Corollary 2.5 Let $\Gamma$ be a regular graph with eigenvalues $\lambda_{0}>\lambda_{1}>\cdots>\lambda_{d}$ and alternating polynomial $P$. Then $\Gamma$ is an $(n, k, c)$-strongly distance-regular graph if and only if any of the following conditions hold.

(a) The harmonic mean $H$ of the numbers $s_{d-1}(u), u \in V$, is

$$
H=\frac{n P\left(\lambda_{0}\right)}{P\left(\lambda_{0}\right)^{2}+n-1} \text {. }
$$

(b) The distance-d graph $\Gamma_{d}$ is $k$-regular with degree

$$
k=\frac{n(n-1)}{P\left(\lambda_{0}\right)^{2}+n-1} .
$$

In such a case, the other parameters of $\Gamma_{d}$ are $(a=) c=k(k-1) /(n-1)$. 
Proof. We only prove sufficiency for condition (a), the other reasonings being almost straightforward from previous material. Note that the right-hand expression in (25) corresponds to the value of the function $\Psi$ at $t=0$ satisfying

$$
\Psi(0) \leq \Psi\left(t_{0}\right)=\Psi_{\max } \leq H .
$$

Then, if equality (25) holds, it must be $t_{0}=0, \Psi_{\max }=H$ and, by Theorem 2.2, $\Gamma$ is strongly distance-regular. Moreover, for such a value of $t_{0}$, and using that $P\left(\lambda_{0}\right)=2 \Sigma_{e}+1=2 \Sigma_{o}-1$ and $\sigma_{o}=n-\sigma_{e}-1$, Eq. (16) yields

$$
\frac{\sigma_{o}}{\sigma_{e}} \frac{P\left(\lambda_{0}\right)-1}{P\left(\lambda_{0}\right)+1}=\frac{\sigma_{o}}{\sigma_{e}} \frac{\Sigma_{e}}{\Sigma_{o}}=1 \Rightarrow \frac{\Sigma_{e}}{\sigma_{e}}=\frac{\Sigma_{o}}{\sigma_{o}} \quad \text { and } \quad \sigma_{e}=\frac{(n-1)\left(P\left(\lambda_{0}\right)-1\right)}{2 P\left(\lambda_{0}\right)}
$$

whence (13) gives

$$
a=c=k-k^{2} \frac{\Sigma_{e}^{2}}{\sigma_{e}^{2}}=k-k^{2} \frac{P\left(\lambda_{0}\right)^{2}}{(n-1)^{2}}=\frac{k(k-1)}{n-1}
$$

(where we have used that $P\left(\lambda_{0}\right)^{2}=\left(\frac{n}{k-1}-1\right)(n-1)$ ), as claimed.

Notice that, as before, the characterization in (a) implies that of (b). The latter was given in [5] for diameter $d=3$, whereas the general case was solved in [11].

Let us now consider the antipodal case. Note that a distance-regular graph $\Gamma$ is an antipodal $r$-cover if and only if its distance- $d$ graph $\Gamma_{d}$ is constituted by disjoint copies of the complete graph $K_{r}$, which can be seen as a (unconnected) $(n, r-1 ; r-2,0)$ strongly regular graph.

Corollary 2.6 Let $\Gamma$ be a regular graph with $n$ vertices, eigenvalues $\lambda_{0}>\lambda_{1}>\cdots>$ $\lambda_{d}, \Sigma=\sum_{i=0}^{d}\left(\pi_{0} / \pi_{i}\right)$, and sum of even multiplicities $\sigma_{e}$. Then $\Gamma$ is an $r$-antipodal distance-regular graph if and only if $\sigma_{e}=\Sigma_{e}$ and the harmonic mean of the numbers $s_{d-1}(u), u \in V$, is

$$
H=n\left(1-\frac{2}{\Sigma}\right)-1
$$

In this case, $r=2 n / \Sigma$.

Proof. Again we only prove sufficiency. Let $\Sigma=P\left(\lambda_{0}\right)+1$. With $\sigma_{e}=\Sigma_{e}$, Eq. (18) particularizes to

$$
\Psi_{\max }=n-\frac{\sigma_{o}}{\Sigma_{o}}=n-\frac{n-\Sigma_{e}-1}{\Sigma_{o}}=n-\frac{2 n}{\Sigma}-1,
$$

where we have used that $\Sigma_{o}=\Sigma_{e}+1=\Sigma / 2$. Hence, by Theorem $2.2, \Gamma$ is strongly distance-regular, with $\Gamma_{d}$ having degree $k=(2 n / \Sigma)-1$. Finally, the antipodal character comes from (13), giving $c=0$ and $a=k-1$, so that $r=k+1=2 n / \Sigma$.

A similar result in terms of $r$ can be found in [7] without using the condition on the multiplicity sum $\sigma_{e}=\Sigma_{e}$. 
THE ELECTRONiC JOURnAL OF COMBINATORICs 7 (2000), \#R51

\section{References}

[1] E. Bannai and T. Ito, Algebraic Combinatorics I: Association Schemes. Benjamin/Cummings, Menlo Park, CA, 1984.

[2] N. Biggs, Algebraic Graph Theory. Cambridge University Press, Cambridge, 1974; second edition: 1993.

[3] A.E. Brouwer, A.M. Cohen, and A. Neumaier, Distance-Regular Graphs. Springer-Verlag, Berlin, 1989.

[4] P.J. Cameron, Strongly regular graphs, in: Selected Topics in Graph Theory, edited by L.J. Beineke and R.J. Wilson. Academic Press, New York, 1978, 337360 .

[5] E.R. van Dam, Bounds on special subsets in graphs, eigenvalues and association schemes, J. Algebraic Combin. 7 (1998), no. 3, 321-332.

[6] E.R. van Dam and W.H. Haemers, A characterization of distance-regular graphs with diameter three, J. Algebraic Combin. 6 (1997), no. 3, 299-303.

[7] M.A. Fiol, An eigenvalue characterization of antipodal distance-regular graphs. Electron. J. Combin. 4 (1997), no. 1, \#R30.

[8] M.A. Fiol, Algebraic characterizations of distance-regular graphs, Discrete Math. (Proc. 11th Inter. Conf. on Formal Power Series and Algebraic Combinatorics, Barcelona, 7-11 June 1999), to appear.

[9] M.A. Fiol, Some spectral characterizations of strongly distance-regular graphs. Combin. Probab. Comput., to appear.

[10] M.A. Fiol and E. Garriga, From local adjacency polynomials to locally pseudodistance-regular graphs, J. Combin. Theory Ser. B 71 (1996), 162-183.

[11] M.A. Fiol and E. Garriga, Pseudo-strong regularity around a set, submitted.

[12] M.A. Fiol, E. Garriga, and J.L.A. Yebra, On a class of polynomials and its relation with the spectra and diameters of graphs, J. Combin. Theory Ser. B 67 (1996), 48-61.

[13] M.A. Fiol, E. Garriga and J.L.A. Yebra, Locally pseudo-distance-regular graphs, J. Combin. Theory Ser. B 68 (1996), 179-205.

[14] M.A. Fiol, E. Garriga, and J.L.A. Yebra, From regular boundary graphs to antipodal distance-regular graphs, J. Graph Theory 27 (1998), no. 3, 123-140.

[15] C.D. Godsil, Algebraic Combinatorics. Chapman and Hall, London/New York, 1993.

[16] W.H. Haemers, Distance-regularity and the spectrum of graphs, Linear Algebra Appl. 236 (1996), 265-278. 